\title{
A Spectral Finite Difference Method for Analysis of a Fluid-Lubricated Herringbone Grooves Journal Bearing under a Special Case at Rectangle Groove
}

\author{
Jun Liu \\ Tochigi R\&D Center, Keihin-Corp.,Tochigi, Japan \\ Email:liujun3@hotmail.com
}

How to cite this paper: Liu, J. (2019) A Spectral Finite Difference Method for Analysis of a Fluid-Lubricated Herringbone Grooves Journal Bearing under a Special Case at Rectangle Groove. Applied Mathematics, 10, 1029-1038.

https://doi.org/10.4236/am.2019.1012071

Received: October 14, 2019

Accepted: December 8, 2019

Published: December 11, 2019

Copyright $\odot 2019$ by author(s) and Scientific Research Publishing Inc. This work is licensed under the Creative Commons Attribution International License (CC BY 4.0).

http://creativecommons.org/licenses/by/4.0/

(c) (i) Open Access

\begin{abstract}
A spectral difference method is applied to get numerical solutions for a fluid-lubricated herringbone grooved journal bearing with trapezoidal grooves by previous work of the authors. However, an inexpedience in which Fourier series of the film profile does not converge at jump points of groove start or groove end in the case of rectangle groove was still remained. In the paper, an inexpedience of numerical analysis under a special case at rectangle groove is challenged to solve. As a result, for compensation of which Fourier series does not converge at jump points in a special case of rectangle groove, Fourier coefficient of fluid film thickness is proposed as taking the limit of which in a case trapezoidal groove at trapezoidal angle approaches 0 .
\end{abstract}

\section{Keywords}

Fluid-Lubricated, Herringbone Grooves, Journal Bearing, Spectral Difference Scheme, Fourier Series, Gibbs Phenomenon

\section{Introduction}

Recently, herringbone grooves journal bearing is used extensively in small high-speed rotating mechanisms, due to their outstanding higher stability and lower leakage, in comparison with a plain journal bearing.

Until now, numerical studies are carried out to investigate characteristics of herringbone groove journal bearing by many researchers. Vohr and Pan [1] proposed narrow groove theory for analysis of a herringbone grooved gas journal bearing in 1963, subsequently applied by Vohr and Chow [2] to get numeri- 
cal solutions at small eccentricity. In 1971, Hamrock and Fleming [3] determined the optimal parameters for self-acting herringbone grooved journal bearings at the maximum radial load capacity.

In 1980, the film in an incompressible fluid was analyzed numerically by $\mathrm{Mu}$ rata et al. [4] based on a potential flow theory. In 1984, Kawabata, Ashino, and Tachibana [5] treated a case of large eccentricity, using a narrow groove theory. In 1994, Bonneau and Absi [6] applied a finite element method to a compressible Reynolds equation to get aerodynamic characteristics for 4 through 16 grooves with moderate eccentricity.

In 2010, the authors employed a spectral difference scheme to analyze an oil-lubricated herringbone grooved journal bearing with trapezoidal groove [7]. The fluid film thickness with trapezoidal groove geometry is expressed in a Fourier series to the circumferential direction, and Reynolds equation is decomposed into each component of the Fourier series, so that it can be integrated with respect to time independently of each component to get a steady-state solution [8] [9]. This process results in high resolution in space and high speed in computation. However, an inexpedience associated with Fourier series expansion of fluid film thickness has be faced at the case of rectangle groove-the persistent discrepancy, an overshoot (Gibbs phenomenon) between fluid film thickness and its approximation by a Fourier series at the number of terms in the series becomes indefinitely large. In the paper, an inexpedience of numerical analysis under a special case at rectangle groove is challenged to solve, and the spectral difference scheme will be extended as to be valid under a special case at rectangle groove.

\section{Analytical Model}

Consider a fluid-lubricated journal bearing equipped with herringbone grooves as shown in Figure 1. Let bearing length be 21 and groove be symmetric with respect to its center of bearing. The shaft itself rotates around its center $\mathrm{O}_{s}$ with an angular velocity $\omega$ in the counter-clockwise direction, and revolves around the center $\mathrm{O}_{b}$ of the fixed-bearing with an angular velocity $\Omega$ in the counter-clockwise direction, the inner radius of the bearing is $R_{b 0}$, the bearing clearance $C_{r}$ is defined as $C_{r}=R_{b 0}-R_{s 0}$, and the radius of the shaft corresponding to the plane without grooves is $R_{50}$, the groove depth, the groove width, ridge width, and grooves angle are denoted by $C_{g}, a_{g}, a_{r}$, and $\beta$ respectively.

The eccentricity of the shaft is given by $\overline{\mathrm{O}_{\mathrm{b}} \mathrm{O}_{\mathrm{s}}}=e$, and the fluid film thickness, $h$, is defined as $h \equiv R_{b}-R_{s}$.

Here two coordinate systems $(\theta, z)$ and $(\zeta, \varphi)$ which are fixed at the rotation shaft, are introduced as shown in Figure 2, and relationship between the coordinates is given by

$$
\begin{gathered}
\theta=\zeta+\varphi \cos (\beta), \\
z=\varphi \sin (\beta) .
\end{gathered}
$$




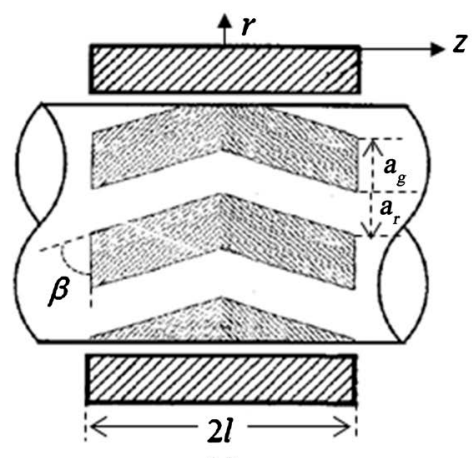

(a)

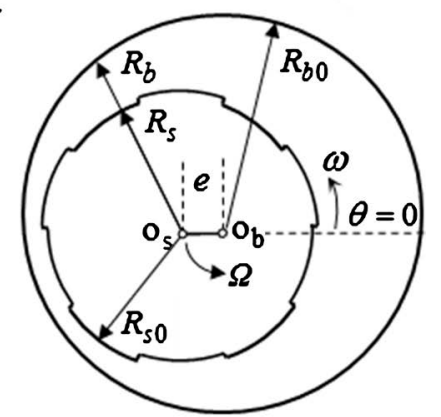

(b)

Figure 1. Herringbone-grooved journal bearing.

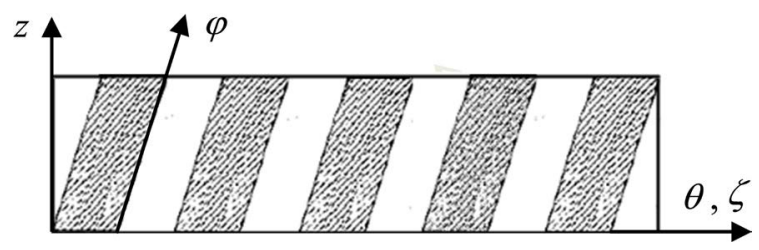

Figure 2. Coordinate systems $(\theta, z)$ and $(\zeta, \varphi)$.

\section{A Spectral Finite Difference Scheme for Reynolds Fluid Lubrication Equation}

In coordinate system $(\zeta, \varphi)$, assuming a steady state, $e=$ const and $\Omega=0$, the dimensionless Reynolds equation can be written as

$$
\nabla_{\Phi}\left(H^{3} \nabla_{\Phi} P\right)+\nabla_{\Psi}\left(H^{3} \nabla_{\Psi} P\right)=-6 \sigma V_{s} \nabla_{\Phi} H-6 \sigma H \nabla_{\Phi} V_{s},
$$

where

$$
\begin{gathered}
\nabla_{\Phi}=\frac{\partial}{\partial \Phi}, \quad \nabla_{\Psi}=-\frac{1}{\tan (\beta)} \frac{\partial}{\partial \Phi}+\frac{1}{\sin (\beta)} \frac{\partial}{\partial \psi} \\
\Phi=\zeta, \quad \Psi=\frac{\varphi}{R_{b 0}}, \quad P=\frac{p}{P_{a}}, \quad H=\frac{h}{R_{b 0}}, \quad \sigma=\frac{\omega \eta}{p_{a}}
\end{gathered}
$$

the dimensionless velocities, $V_{s}$, at the surface of the rotating shaft are given by

$$
V_{s} \equiv \frac{R_{s} \omega}{R_{b 0} \omega}=R_{s / b} .
$$

In a spectral finite difference scheme, the Equation (2b) is decomposed into each component of the Fourier series to the circumferential $\Phi$-direction.

$$
\begin{gathered}
H(\Psi, \Phi)=\sum_{n=0}^{\infty} H_{c n}(\Psi) \cos (n \Phi)+\sum_{n=1}^{\infty} H_{s n}(\Psi) \sin (n \Phi), \\
P(\Psi, \Phi)=\sum_{n=0}^{\infty} P_{c n}(\Psi) \cos (n \Phi)+\sum_{n=1}^{\infty} P_{s n}(\Psi) \sin (n \Phi),
\end{gathered}
$$

at $\Psi=0$, the groove shape is symmetric, the boundary conditions of pressure are

$$
\frac{\partial P_{c n}}{\partial \Psi}=n P_{s n} \cos (\beta) \quad(n \geq 0)
$$




$$
\frac{\partial P_{s n}}{\partial \Psi}=-n P_{c n} \cos (\beta) \quad(n \geq 1),
$$

and it is assumed that the fluid is open to the atmosphere at $\Psi=L / \tan \beta$, so that boundary conditions of pressure are

$$
\begin{gathered}
P_{c n}=1.0 \quad(n=0), \\
P_{c n}=0, P_{s n}=0 \quad(n \geq 1) .
\end{gathered}
$$

\section{Fourier Series of Fluid Film Thickness Geometry}

The dimensionless fluid film thickness of herringbone grooves journal bearing can be rewritten as

$$
H(\Psi, \Phi)=H_{0}(\Phi)+E \cos [\Phi+\Psi \cos (\beta)],
$$

where $H_{0}$, is fluid film thickness without eccentricity, $E$ is dimensionless eccentricity as $E=e / R_{b 0}$.

1) In the Case of Trapezoidal Groove

As for a trapezoidal groove which was assumed as shown in Figure 3, $\Delta \Phi$ is defined as trapezoidal angle of groove.

In case of $a_{r} / a_{g}=1.0$, the fluid film thickness with trapezoidal grooves, $H_{0}$, is given by

$$
H_{0}(\Phi)=\left\{\begin{array}{lc}
C_{r / b} & \text { ridge } \\
C_{r / b}+\frac{C_{g / b}}{\Delta \Phi}\left(\Phi-\Phi_{i}\right) & \text { ridge } \rightarrow \text { groove } \\
C_{r / b}+C_{g / b}-\frac{C_{g / b}}{\Delta \Phi}\left(\Phi-\Phi_{i}\right) & \text { groove } \rightarrow \text { ridge } \\
C_{r / b}+C_{g / b} & \text { groove }
\end{array},\right.
$$

where, $C_{r / b}=\frac{C_{r}}{R_{b 0}}, C_{g / b}=\frac{C_{g}}{R_{b 0}}$.

The Fourier cosine and sine coefficient of fluid film thickness are obtained as

$$
H_{0_{-} c 0}=C_{r / s}+\frac{C_{g / b}}{2},
$$

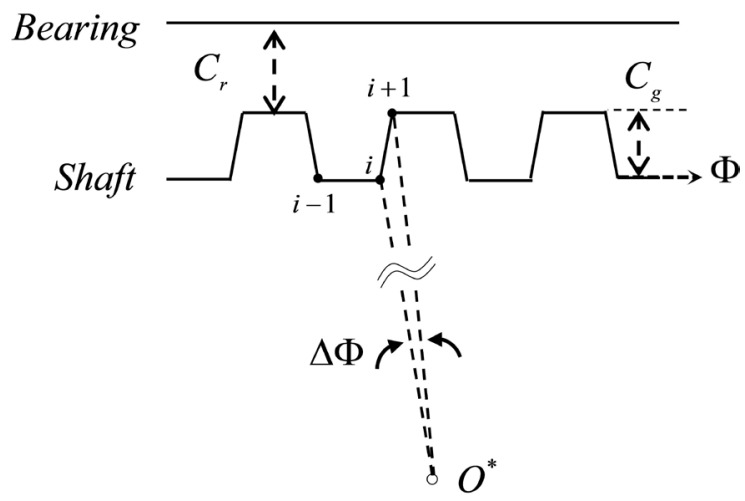

Figure 3. Cross-section perpendicular to the trapezoidal surface of groove. 


$$
\begin{gathered}
H_{0_{-} c n}=\frac{2}{\pi} \frac{C_{g / b}}{n^{2} \Delta \Phi} \sum_{k=1}^{2 N_{g}}\left[(-1)^{k} \sin \left(\frac{n k \pi}{N_{g}}-\frac{n \Delta \Phi}{2}\right) \sin \left(\frac{n \Delta \Phi}{2}\right)\right], \\
H_{0_{-} s n}=-\frac{2}{\pi} \frac{C_{g / b}}{n^{2} \Delta \Phi} \sum_{k=1}^{2 N_{g}}\left[(-1)^{k} \cos \left(\frac{k n \pi}{N_{g}}-\frac{n \Delta \Phi}{2}\right) \sin \left(\frac{n \Delta \Phi}{2}\right)\right],
\end{gathered}
$$

where $N_{g}$ is number of grooves.

The "error" in the partial Fourier series at $n=N$ in case of trapezoidal grooves is given as follows,

$$
\begin{aligned}
\text { error }= & \sum_{n=N+1}^{\infty} H_{c n}(\Psi) \cos (n \Phi)+\sum_{n=N+1}^{\infty} H_{s n}(\Psi) \sin (n \Phi) \\
= & \frac{1}{\pi} \frac{C_{g / b}}{\Delta \Phi} \sum_{k=1}^{2 N_{g}}(-1)^{k}\left\{\frac{\cos \left(\frac{N k \pi}{N_{g}}\right)}{N}-\frac{\cos \left(\frac{N k \pi}{N_{g}}-N \Delta \Phi-N \delta \Phi\right)}{N}\right. \\
& +\Delta \Phi\left[S i\left(\frac{N k \pi}{N_{g}}-N \Delta \delta \Phi\right)\right] \\
& \left.+\left(\frac{k \pi}{N_{g}}-\delta \Phi\right)\left[S i\left(\frac{N k \pi}{N_{g}}-N \delta \Phi\right)-S i\left(\frac{N k \pi}{N_{g}}-N \Delta \Phi-N \delta \Phi\right)\right]\right\} \\
= & \frac{1}{\pi} \frac{C_{g / b}}{\Delta \Phi} \sum_{k=1}^{2 N_{g}}(-1)^{k}\left\{-\frac{2}{N} \sin \left(\frac{N k \pi}{N_{g}}-\frac{N \Delta \Phi}{2}\right) \sin \frac{N \Delta \Phi}{2}+\Delta \Phi\left[S i\left(\frac{N k \pi}{N_{g}}\right)\right]\right. \\
& +\left(\frac{k \pi}{N_{g}}\right)\left[S i\left(\frac{N k \pi}{N_{g}}\right)-S i\left(\frac{N k \pi}{N_{g}}-N \Delta \Phi\right)\right]
\end{aligned}
$$

where $\operatorname{Si}(x)$ is sinc function. Figure 4 plot the maximum error in case of $N_{g}=$ 5 with the partial Fourier series term number increases, and obviously, the maximum error decays rapidly in case of trapezoidal grooves.

2) In a Special Case at Rectangle Groove

More generally, for the Fourier series expansion of fluid film thickness in case of rectangle groove, the nth partial Fourier series will overshoot this jump by
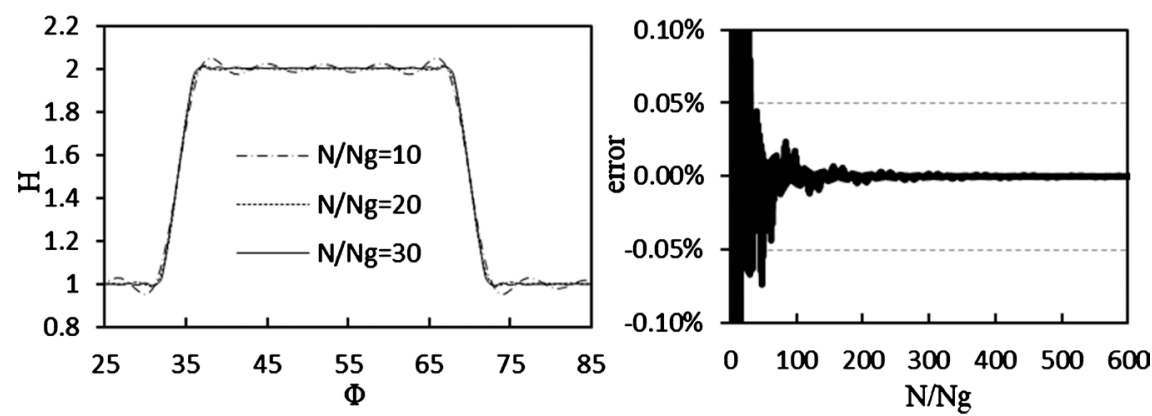

Figure 4. The maximum error with variation of the partial Fourier series term number in case of trapezoidal grooves. 
approximately at a point of groove start or groove end (Gibbs phenomenon), the "error" in the partial Fourier series will be about $8.95 \%$ and $14.11 \%$ of groove depth larger than the jump in the original fluid film thickness in two cases, in the limits of increasing many terms and of increasingly high node densities, respectively, see Figure 5 by Bengt Fornberg [10].

How to reduce the Gibbs phenomenon, is an interesting and important topic in mathematics, several mathematician deal with this topic, and obtained some method for its field, e.g. filtering and spectral re-projection. Filtering is a classical tool for mitigating the Gibbs phenomenon in Fourier expansions, however filtering does not completely remove the Gibbs phenomenon. To completely remove the Gibbs phenomenon, one can re-expand the function in a carefully chosen different basis, it is spectral re-projection method, which is given by Gottlieb and Shu [11]. In 1990, Gottlieb, together with Shu and other co-works, showed that it is possible to completely remove the Gibbs phenomenon by post-processing the Fourier expansion in regions in which the function is analytic, using a re-expansion of the partial sums $f_{N}(x)$ in a different basis ("Gibbs complementary"). But understanding that needs high mathematic knowledge, and it is not easy for industrial applications, an easy analysis method always requested from engineer, and so for compensation of its defect, the following method which is proposed.

Since Equation (8) are continuity and differentiable at a point $\Delta \Phi=0$, then Fourier coefficient of fluid film thickness in the case of rectangle groove can been replaced as taking the limit of Equation (8) at trapezoidal angle $\Delta \Phi$ approaches 0 , which are given as

$$
\begin{gathered}
\lim _{\Delta \Phi \rightarrow 0} H_{0_{-} c 0}=C_{r / b}+\frac{C_{g / b}}{2}, \\
\lim _{\Delta \Phi \rightarrow 0} H_{0_{-} c n}=\frac{C_{g / b}}{\pi n} \sum_{k=1}^{2 N_{g}}\left[(-1)^{k} \sin \left(\frac{n k \pi}{N_{g}}\right)\right],
\end{gathered}
$$

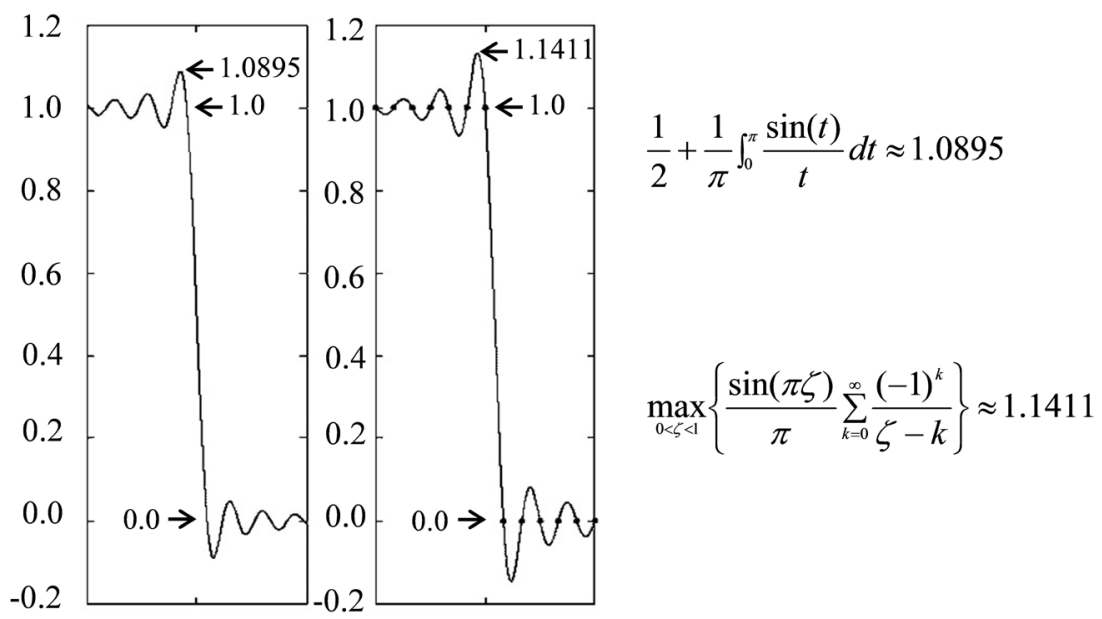

Figure 5. The Gibbs Phenomenon for Fourier series by Bengt Fornberg \& Natasha Flyer (2006). 


$$
\lim _{\Delta \Phi \rightarrow 0} H_{0_{-} s n}=-\frac{C_{g / b}}{\pi n} \sum_{k=1}^{2 N_{g}}\left[(-1)^{k} \cos \left(\frac{n k \pi}{N_{g}}\right)\right],
$$

and the maximum "error" at $n=N$ of proposed method which is Equation (9) can be obtained

$$
\begin{aligned}
\text { error } & =\sum_{n=N+1}^{\infty} H_{c n}(\Psi) \cos (n \Phi)+\sum_{n=N+1}^{\infty} H_{s n}(\Psi) \sin (n \Phi) \\
& \approx \frac{C_{g / b}}{\pi} \sum_{k=1}^{2 N_{g}}(-1)^{k} \int_{N+1}^{\infty} \frac{1}{n}\left\{\sin \left(\frac{n k \pi}{N_{g}}-n \delta \Phi\right)\right\} \mathrm{d} n \\
& =\frac{C_{g / b}}{\pi}\left\{\sum_{k=1}^{2 N_{g}}(-1)^{k} \operatorname{Si}\left(N\left(\frac{k \pi}{N_{g}}-\delta \Phi\right)\right)\right\}
\end{aligned}
$$

and since $k \pi / N_{g} \gg \delta \Phi$, thus

$$
\text { error }=\frac{C_{g / b}}{\pi} \sum_{k=1}^{2 N_{g}}(-1)^{k} S i\left(\frac{N \pi k}{N_{g}}\right) .
$$

Figure 6 plots the maximum error in case of $N_{g}=5$ with the partial Fourier series term number increases, and obviously, the maximum error decays rapidly in case of trapezoidal grooves.

Let shows an example of the film thickness at rectangle groove by Fourier's series using the limit of trapezoidal angle $\Delta \Phi$ approaches 0 in Figure 7 , the "error" in the partial Fourier series in the case of rectangle groove was cut off, and the inexpedience which Fourier series of the film profile does not converge at jump points of groove start or groove end in the case of rectangle groove would be avoided.

\section{Analyzed Examples}

To confirm the applicability of the above method, Hirs model [12] which is $N_{g}$ $=20, \beta=21.8$ deg., $a_{r} / a_{g}=1.0, L=R_{b}, \Omega=0, \Lambda \equiv 6 \sigma \times\left(R_{b} / C_{r}\right)^{2}=0.21$ was picked, the relation between load capacity and eccentricity are numerical analyzed, and which compare to experiments of Hirs as shown in Figure 8, it shows that those numerical results are in good agreement with the experimental data.
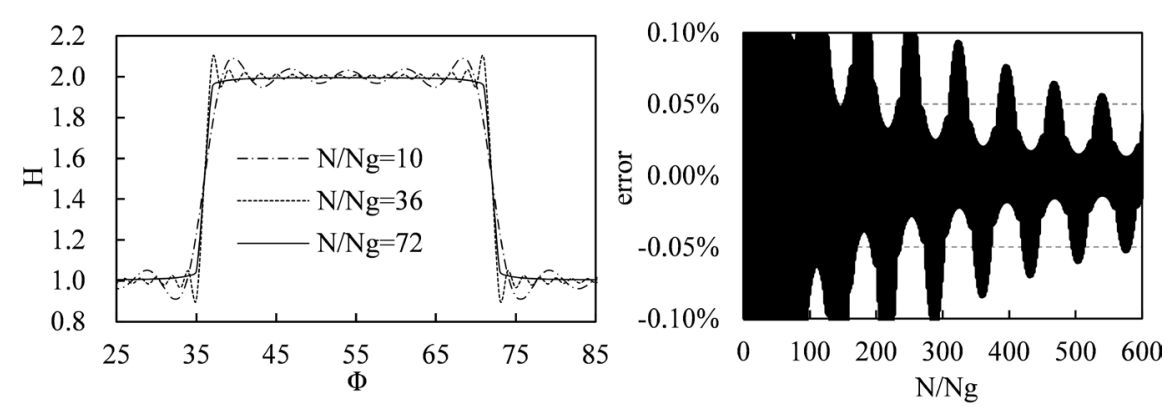

Figure 6. The maximum error with variation of the partial Fourier series term number in case of rectangle grooves. 
trapezoidal groove $\Delta \Phi(=4 \mathrm{deg}.) \rightarrow 0$ rectangle groove

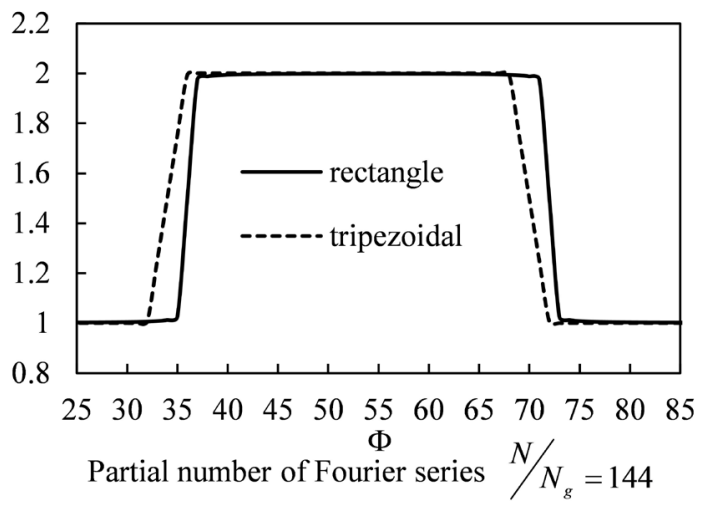

Figure 7. The film surface profile at rectangle groove was displayed using the limit of trapezoidal angle $\Delta \Phi$ approaches 0 .

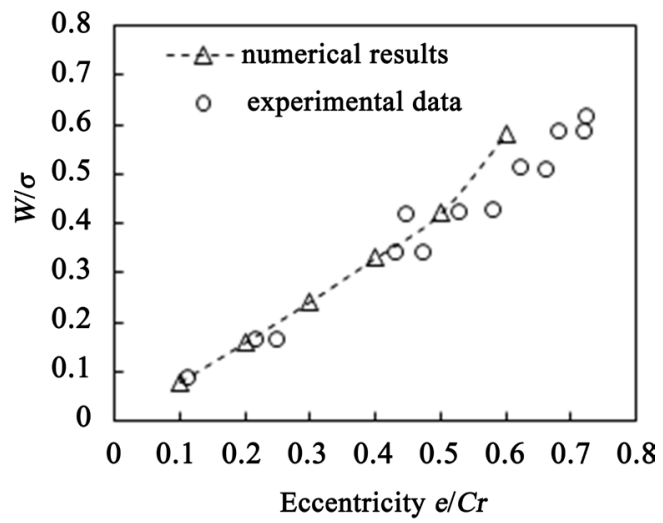

Figure 8. Variations of load capacity with eccentricity increases, where, $W$ is the dimensionless load capacity.

\section{Conclusions}

As analysis of a fluid-lubricated herringbone grooved journal bearing under a spectral difference scheme, for compensation of which Fourier series does not converge at jump points in a special case of rectangle groove. Fourier coefficient of fluid film thickness is proposed as taking the limit of which in a case trapezoidal groove at trapezoidal angle approaches 0 . In addition, the difference of the film thickness and the number of terms of its Fourier series are investigated with the ratio of the Fourier's series terms number to grooves number.

A spectral difference method is applied to get numerical solutions for a fluid-lubricated herringbone grooved journal bearing with trapezoidal grooves, and then the numerical analysis scheme will be extended to be suitable for a special case of rectangle groove.

Fourier coefficients of fluid film can be replaced as taking the limit of the trapezoidal angle approaches 0 .

\section{Acknowledgements}

The author gratefully acknowledges Emeritus Professor Y. Mochimaru of the 
Tokyo Institute of Technology for advice.

\section{Conflicts of Interest}

The author declares no conflicts of interest regarding the publication of this paper.

\section{References}

[1] Vohr, J.H. and Pan, C.H.T. (1963) On the Spiral-Grooved, Self-Acting Gas Bearing. MTI63TR52.

[2] Vohr, J.H. and Chow, C.Y. (1965) Characteristics of Herringbone Egrooved, Gas-Lubricated Journal Bearings. Journal of Fluids Engineering, 87, 568-578. https://doi.org/10.1115/1.3650607

[3] Hamrock, B.J. and Fleming, D.P. (1971) Optimization of Self-Acting Herringbone Grooved Journal Bearings for Maximum Radial Load Capacity. NASA-TM-X-52945.

[4] Murata, S., Miyake, Y. and Kawabata, N. (1980) Two-Dimensional Analysis of Herringbone Groove Journal Bearing. Bulletin of the JSME, 23, 1980-1987.

https://doi.org/10.1299/jsme1958.23.1220

[5] Kawabata, N., Ashino, I., Tachibana, M., et al. (1983) Analysis of the Load Carrying Capacity of Herringbone-Grooved Journal Bearings. Transactions of the JSME, 49, 648-656. (In Japanese)

[6] Bonneau, D. and Absi, J. (1994) Analysis of Aerodynamic Journal Bearing with Small Number of Herringbone Grooves by Finite Element Method. Journal of Tribology, 116, 698-704. https://doi.org/10.1115/1.2927320

[7] Liu, J. and Mochimaru, Y. (2010) Analysis of Oil-Lubricated Herringbone Grooved Journal Bearing with Trapezoidal Cross-Section, Using a Spectral Finite Difference Method. Journal of Hydrodynamics, 22, 408-412. https://doi.org/10.1016/S1001-6058(09)60228-6

[8] Liu, J. and Mochimaru, Y. (2012) The Effects of Trapezoidal Groove on a Self-Acting Fluid-Lubricated Herringbone Grooves Journal Bearing. ISRN Tribology, 2013, Article ID: 240239. https://doi.org/10.5402/2013/240239

[9] Liu, J. and Mochimaru, Y. (2012) Pressure Distributions Generated along a Self-Acting Fluid-Lubricated Herringbone-Grooved Journal Bearing with Trapezoidal Groove. ISRN Tribology, 2013, Article ID: 560917. https://doi.org/10.5402/2013/560917

[10] Fornberg, B. and Flyer, N. (2007) The Gibbs Phenomenon for Radial Basis Functions. In: Jerri, A., Ed., The Gibbs Phenomenon in Various Representations and Applications, Sampling Publishing, Potsdam, NY, 201-224

[11] Gottlieb, D. and Shu, C.W. (1994 ) On The Gibbs Phenomenon V: Recovering Exponential Accuracy in a Sub-Interval from a Gegenbauer Partial Sum of a Piecewise Analytic Function. NASA Contractor Report 194952, ICASE Report No. 94-61.

[12] Hirs, G.G. (1965) The Load Capacity and Stability Characteristics of Hydro-Dynamic Grooved Journal Bearing. ASLE Transactions, 8, 296-305.

https://doi.org/10.1080/05698196508972102 


\section{Nomenclature}

$a_{g}, a_{r}=$ grooves width, ridge width

$C_{g}, C_{g / b}=$ groove depth, dimensionless groove depth

$C_{r}=$ bearing clearance

$e, E=$ eccentricity, dimensionless eccentricity

$H=$ dimensionless fluid film thickness

l, $L=$ bearing length, dimensionless bearing length

$N_{g}=$ number of grooves

$p, P=$ pressure, dimensionless pressure

$P_{a}=$ atmospheric pressure

$r, \theta, z=$ inertial coordinates

$R_{b}=$ radius of bearing

$R_{s}=$ radial component of coordinate at surface of shaft

$R_{s 0}=$ radius of shaft without grooves

$R_{s / b}=$ dimensionless radial component of coordinate at surface of shaft

$t, \tau=$ time, dimensionless time

$u_{s}=$ circumferential velocity at surface of rotating shaft

$U_{s}=$ dimensionless circumferential velocity at surface of rotating shaft

$V_{s}=$ radial velocity at surface of rotating shaft

$V_{s}=$ dimensionless radial velocity at surface of rotating shaft

$v_{r}, v_{\theta}, v_{z}=$ velocity components of lubricant fluid

$W=$ dimensionless load capacity of bearing

$\beta=$ groove angle

$\eta=$ viscosity of fluid

$\Lambda=$ bearing number

$\sigma=$ dimensionless number

$\varphi=$ attitude angle of shaft

$\Delta \Phi=$ trapezoidal angle of groove

$\phi, \Phi=$ angle between the fixed axis of abscissa $(\theta=0)$ and the axis of eccentric-

ity, dimensionless angle

$\omega=$ rotation velocity of shaft

$\Omega=$ swirl velocity of shaft

superscript $^{\star}$ : non-inertial coordinate 